\title{
Combined Temporal and Spatial Filter Structures for CDMA Systems
}

\author{
Aylin Yener \\ WINLAB, Rutgers University \\ yener@winlab.rutgers.edu
}

\author{
Roy D. Yates \\ WINLAB, Rutgers University \\ ryates@winlab.rutgers.edu
}

\author{
Sennur Ulukus \\ AT\&T Labs-Research \\ ulukus@research.att.com
}

\begin{abstract}
CDMA systems are interference limited and therefore efficient interference management is necessary to enhance the capacity of a CDMA system. In this paper, we consider combining two effective receiver based interference management strategies: multiuser detection (temporal filtering) and receiver beamforming (spatial filtering). We formulate and examine the performance of several linear filter structures which are all based on minimum mean squared error (MMSE) criteria, but differ in how the MMSE problems are defined in the temporal and spatial domains, i.e., jointly or in cascade. It is shown that while the joint optimum MMSE filter achieves the maximum signal-to-interference ratio (SIR) among all possible linear filters, the constrained optimum MMSE filter which results in a single temporal and single spatial filter, outperforms all combined singleuser/multiple-user approaches and cascaded optimization approaches either uniformly or asymptotically. The constrained optimum MMSE filter is near-far resistant in all but very highly loaded systems and enjoys low complexity.
\end{abstract}

\section{Introduction}

The demand for high capacity flexible wireless services is ever-growing. CDMA shows promise in meeting this demand and consequently W-CDMA [1] has been proposed as a standard for the third-generation (3G) wireless systems. It is well known that CDMA systems are interference limited and suffer from near-far effect. The challenge to enhance the capacity of a CDMA system therefore lies in interference management. In this work, we concentrate on the two commonly used interference management methods: multiuser detection and receiver beamforming. Both methods aim at suppressing or cancelling the interference using receiver signal processing; multiuser detection exploits the temporal structure whereas beamforming exploits the spatial structure of the interference for interference management $[7,12]$.

Multiuser detection [12] performs temporal filtering of the received signal to effectively suppress the multiple ac- cess interference. The optimum multiuser detector has been shown to be exponentially complex in the number of users, and a number of low complexity suboptimum receivers have been proposed following this development $[5,6]$. Increasing the capacity of CDMA systems by employing antenna arrays at the base station has been proposed in [8], where the outputs of the multiple antenna array elements are combined to make bit decisions for the user. In [8], matched filter receivers are assumed in the temporal domain for each user and the array observations are combined via a filter that is matched to the array response of the user, i.e., single user processing is employed in both domains.

Another method of capacity enhancement which utilizes the spatial diversity is space-time processing for CDMA which traditionally refers to receiver beamforming (space processing) and multipath combining (time processing) [9]. The received signals from different paths and antennas are combined to better decode the desired user's bits. However, the inherent structure of the multiple access interference is not exploited, i.e., no multiuser detection is employed $[2,4,14]$. A recent paper [13] addresses the derivation of the sufficient statistics and the optimum and some linear suboptimum multiuser detectors when an antenna array is present at the receiver for a multipath channel.

In this work, we will investigate the possible receiver filter structures when both multiuser detection and beamforming are employed to further increase the uplink capacity of a CDMA system. Linear processing is assumed in both the temporal and the spatial domains and the temporal-spatial filters are denoted by two-dimensional matrix filters. Within this framework, there are several possible filter structures. One can derive the jointly optimal temporal and spatial filter that minimizes the mean squared error (MSE) between the information bit and the filter output of a desired user. Since this joint MMSE filter may have high computational complexity, less complex filters which nevertheless provide efficient interference suppression are of interest. To serve this purpose, recently, constrained optimum filters are proposed by forcing the joint receiver filter matrix to be of rank $1[15,16]$. One can also construct cascaded filters where MSE optimization is carried over in temporal and spatial 
domains independently in tandem, in both spatial-temporal and temporal-spatial orders. While the cascaded spatialtemporal filter is similar to the idea of cascade optimumspace/optimum-time combiner proposed in [2], the temporal combiner in our case is a chip combiner that exploits the temporal structure of the interference composed of the temporal signatures of the interferers, as opposed to a multipath combiner in [2] which is a single user temporal processor. We consider all above filter structures and then give analytical performance comparisons among them. While the joint domain MMSE filter is clearly the SIR maximizing temporal and spatial processor over all matrix filters, an interesting observation is that the constrained optimum temporal spatial processor outperforms all combined single user/multiple user approaches and the cascaded approaches either uniformly or asymptotically.

\section{System Model}

We consider a single cell DS-CDMA system where each user is assigned a unique signature sequence. For clarity of exposition, we assume a synchronous system with processing gain $G$. At the base station, an antenna array of $K$ elements is employed. The received signal at the output of the antenna array at the base station is:

$$
\mathbf{r}(t)=\sum_{j=1}^{N} \sqrt{p_{j}} b_{j} s_{j}(t) \mathbf{a}_{j}+\mathbf{n}(t)
$$

where $p_{j}, b_{j}, s_{j}(t)$ and $\mathbf{a}_{j}$ are the received power, the bit, the (temporal) signature and the array response vector (spatial signature) of user $j$, respectively. Both the temporal and spatial signatures of the users have unit energy, and the temporal signatures are of the following form

$$
s_{j}(t)=\sum_{l=1}^{G} s_{j}^{(l)} \psi\left(t-(l-1) T_{c}\right)
$$

where $\psi(t)$ is the chip waveform, $T_{c}$ is the chip duration, and $s_{j}^{(l)}= \pm 1 / \sqrt{G}$. Chip matched filtering and sampling the received signal $\mathbf{r}(t)$ at the $l$ th chip interval, we obtain a $K$-dimensional observation vector $\mathbf{r}_{l}$

$$
\mathbf{r}_{l}=\sum_{j=1}^{N} \sqrt{p_{j}} b_{j} \mathbf{a}_{j} s_{j}^{(l)}+\mathbf{n}_{l}
$$

which represents the chip matched filtered samples at the $l$ th chip interval across the $K$ antenna array elements. Over one bit period, $T_{b}=G T_{c}$, we collect a set of $G K$-dimensional vectors $\left\{\mathbf{r}_{l}, 1 \leq l \leq G\right\}$ which we can arrange in a $G \times K$ $\operatorname{matrix} \mathbf{R}=\left\{\mathbf{r}_{1}, \mathbf{r}_{2}, \cdots, \mathbf{r}_{G}\right\}^{\top}$ :

$$
\mathbf{R}=\sum_{j=1}^{N} \sqrt{p_{j}} b_{j} \mathbf{s}_{j} \mathbf{a}_{j}^{\top}+\mathbf{N}
$$

These $K G$ observation samples can also be seen as a collection of $K \quad G$-dimensional vectors, i.e., $\mathbf{R}=\left[\mathbf{z}_{1}, \mathbf{z}_{2}, \cdots, \mathbf{z}_{K}\right]$, where $\mathbf{z}_{k}$ denotes the observation vector consisting $G$ chip matched filtered samples at the output of the $k$ th antenna element and is expressed as

$$
\mathbf{z}_{k}=\sum_{j=1}^{N} \sqrt{p_{j}} b_{j} s_{j} a_{j}^{(k)}+\mathbf{n}_{k}
$$

In (4), $\mathbf{N}$ is the matrix that represents the spatially and temporally white noise, i.e., $E\left[N_{k l}^{*} N_{m n}\right]=\sigma^{2} \delta_{k m} \delta_{l n}$, where $(\cdot)^{*}$ denotes the conjugate of a complex number. We label user $i$ as the desired user and the other users as interferers.

\section{Filter Structures}

The detection of the information bit of the desired user is done by taking the sign of the real part of the decision statistic which is found by combining the entries of the observation matrix $\mathbf{R}$ by using a matrix filter $\mathbf{X}_{i}$. Thus, the decision statistic, $y_{i}$, is the output of a two dimensional linear filter $\mathbf{X}_{i}$ :

$$
y_{i}=\sum_{j=1}^{G} \sum_{l=1}^{K}\left[X_{i}\right]_{j l}^{*} R_{j l}=\operatorname{tr}\left(\mathbf{X}_{i}^{H} \mathbf{R}\right)
$$

where $\operatorname{tr}(\cdot)$ and $(\cdot)^{H}$ are the trace and the hermitian transpose operations, respectively.

In what follows, we investigate the possible filter structures. The filter structures in Sections 3.1, 3.2, and 3.3 use single user processing in at least one of the temporal and spatial domains and are well-known. Next we derive two temporal-spatial filters, the filter structures in Sections 3.4 and 3.5, which use cascaded MMSE optimizations in spatial and temporal domains. The structure of these receivers is to combine either the received chip samples at the output of each array element in the MMSE sense followed by a spatial filter that combines the resulting vector in the MMSE sense (Section 3.4); or to combine all array outputs for each chip sample in the MMSE sense followed by the temporal MMSE combiner for the resulting vector (Section 3.5).

Next, in Sections 3.6 and 3.7, we give the joint temporalspatial MMSE filter structures. The difference between the two joint MMSE structures is the fact that while the joint optimum temporal-spatial MMSE in Section 3.6 is the best filter in terms of minimizing the MSE (and maximizing the SIR) over all possible matrix filters, the constrained optimum temporal-spatial filter in Section 3.7 is the MMSE filter when the filter space is constrained to contain matrix filters of rank 1 only. The physical interpretation of this mathematical constraint on the matrix filter is that it results in a separable filter with a single temporal and a single spatial 
combiner. The difference between the joint MMSE structures in Sections 3.6 and 3.7 and the cascaded MMSE structures in Sections 3.4 and 3.5 lies in the fact that the cascaded structures use temporal and spatial filters that are optimized independently in each domain while the joint structures are found by optimization in both domains simultaneously.

\subsection{Single User Temporal-Spatial Detector}

This is a single user based approach for both the spatial [8]. The decision statistic in this case is $y_{i}=\mathbf{s}_{i}^{\top} \mathbf{R} \mathbf{a}_{i}^{*}=$ $\operatorname{tr}\left(\mathbf{a}_{i}^{*} \mathbf{s}_{i}^{\top} \mathbf{R}\right)$ leading to

$$
\mathrm{X}_{i}^{\mathrm{MF}-\mathrm{MF}}=\mathbf{s}_{i} \mathrm{a}_{i}^{\top}
$$

\subsection{Temporal MMSE Filter-Spatial MF}

This approach uses multiuser processing in temporal domain [6] and single user processing in spatial domain. The decision statistic in this case is $y_{i}=\mathbf{c}_{i}^{\top} \mathbf{R} \mathbf{a}_{i}^{*}=\operatorname{tr}\left(\mathbf{a}_{i}^{*} \mathbf{c}_{i}^{\top} \mathbf{R}\right)$ leading to

$$
\mathbf{X}_{i}^{\mathrm{MMSE-MF}}=\mathbf{c}_{i} \mathbf{a}_{i}^{\top}
$$

where

$$
\mathbf{c}_{i}=\sqrt{p_{i}}\left(\sum_{j=1}^{N} p_{j}\left|\mathbf{a}_{i}^{H} \mathbf{a}_{j}\right|^{2} \mathbf{s}_{j} \mathbf{s}_{j}^{\top}+\sigma^{2} \mathbf{I}\right)^{-1} \mathbf{s}_{i}
$$

\subsection{Temporal MF-Spatial MMSE Filter}

This approach uses single user processing in the temporal domain combined with multi user processing in spatial domain [7]. The decision statistic in this case is $y_{i}=$ $\mathbf{s}_{i}^{\top} \mathbf{R} \mathbf{w}_{i}^{*}=\operatorname{tr}\left(\mathbf{w}_{i}^{*} \mathbf{s}_{i}^{\top} \mathbf{R}\right)$ leading to

$$
\mathbf{X}_{i}^{\text {MF-MMSE }}=\mathbf{s}_{i} \mathbf{w}_{i}^{\top}
$$

where

$$
\mathbf{w}_{i}=\sqrt{p_{i}}\left(\sum_{j=1}^{N} p_{j}\left(\mathbf{s}_{i}^{\top} \mathbf{s}_{j}\right)^{2} \mathbf{a}_{j} \mathbf{a}_{j}^{H}+\sigma^{2} \mathbf{I}\right)^{-1} \mathbf{a}_{i}
$$

\subsection{Cascaded Temporal-Spatial MMSE Filter}

Assume that at the output of each antenna array we are allowed to design a separate temporal filter, i.e., a linear chip combiner. Recall that the output of the $k$ th antenna element is $\mathbf{z}_{k}$. We design $K$ temporal filters $\mathbf{c}_{k}, k=1, \ldots, K$, such that each of the $K$ resulting statistics

$$
\tilde{y}_{k}=\mathbf{c}_{k}^{H} \mathbf{z}_{k}=\sum_{j=1}^{N} \sqrt{p_{j}} b_{j} \mathbf{c}_{k}^{H} \mathbf{s}_{j} a_{j}^{(k)}+\mathbf{c}_{k}^{H} \mathbf{n}_{k}
$$

has minimum mean squared difference from the desired bit, $b_{i}$. The solution can be found as [6]:

$$
\mathbf{c}_{k}=\sqrt{p_{i}} a_{i}^{(k)}\left(\sum_{j=1}^{N}\left|a_{j}^{(k)}\right|^{2} p_{j} \mathbf{s}_{j} \mathbf{s}_{j}^{\top}+\sigma^{2} \mathbf{I}\right)^{-1} \mathbf{s}_{i}
$$

Note that what makes $c_{k}$, the temporal MMSE filter at the output of the $k$ th antenna, different from $c_{l}$, the temporal MMSE filter at the output of the $l$ th antenna, are the different spatial gains users have for different antennas. Defining the modified gain at the output of the $k$ th antenna for user $j$ as $\tilde{a}_{j}^{(k)}=\left(c_{k}^{H} \mathbf{s}_{j}\right) a_{j}^{(k)}$, from (12) we have

$$
\tilde{y}_{k}=\sum_{j=1}^{N} \sqrt{p_{j}} b_{j} \tilde{a}_{j}^{(k)}+\tilde{n}_{k}, \quad k=1, \ldots, K
$$

We can then combine $\left\{\tilde{y}_{k}\right\}$ in the MMSE sense, and similar to (13), express the second stage of the cascaded filter as

$$
\mathbf{w}=\sqrt{p_{i}}\left(\sum_{j=1}^{N} p_{j} \tilde{\mathbf{a}}_{j} \tilde{\mathbf{a}}_{j}^{H}+\mathbf{\Lambda}\right)^{-1} \tilde{\mathbf{a}}_{i}
$$

where $\boldsymbol{\Lambda}=\operatorname{diag}\left\{\sigma^{2}\left(\mathbf{c}_{k}^{H} \mathbf{c}_{k}\right)\right\}$ is the covariance matrix of $\tilde{\mathbf{n}}$. The final bit decision is done by taking the sign of the real part of $y_{i}=\mathbf{w}^{H} \tilde{\mathbf{y}}$.

Note that to construct the overall receiver we need to invert $K G \times G$ matrices and one $K \times K$ matrix. To see how the overall cascaded filter can be expressed as matrix filter $\mathbf{X}_{i}^{\text {TS-CMMSE }}$, observe that

$$
\tilde{y}_{k}=\mathbf{c}_{k}^{H} \mathbf{z}_{k}=\mathbf{c}_{k}^{H} \mathbf{R} \mathbf{e}_{k}=\operatorname{tr}\left(\mathbf{e}_{k} \mathbf{c}_{k}^{H} \mathbf{R}\right)
$$

and

$$
y_{i}=\sum_{k=1}^{K} w_{k}^{*} \tilde{y}_{k}=\operatorname{tr}\left(\sum_{k=1}^{K} w_{k}^{*} \mathbf{e}_{k} \mathbf{c}_{k}^{H} \mathbf{R}\right)
$$

where $e_{k}$ is a $K$ vector which has 1 in its $k$ th entry and zeros elsewhere. Comparing (17) with (6), we find that

$$
\mathbf{X}_{i}^{\mathrm{TS}-\mathrm{CMMSE}}=\sum_{k=1}^{K} w_{k} c_{k} \mathbf{e}_{k}^{\top}
$$

which can be of rank up to $K$.

\subsection{Cascaded Spatial-Temporal MMSE Filter}

Alternatively, one can think of first combining all antenna array elements in each chip interval, followed by a temporal combiner. We first design $G$ spatial filters $w_{l}, l=1, \ldots, G$, to combine the entries of $r_{l}$ in the MMSE sense

$$
\mathbf{w}_{l}=\sqrt{p_{i}} s_{i}^{(l)}\left(\sum_{j=1}^{N}\left(s_{j}^{(l)}\right)^{2} p_{j} \mathbf{a}_{j} \mathbf{a}_{j}^{H}+\sigma^{2} \mathbf{I}\right)^{-1} \mathbf{a}_{i}
$$


Recall from (2) that $\left(s_{j}^{(l)}\right)^{2}=1 / G$, for all $l$. Thus, defining

$$
\hat{\mathbf{w}}=\sqrt{p_{i}}\left(\sum_{j=1}^{N} \frac{1}{G} p_{j} \mathbf{a}_{j} \mathbf{a}_{j}^{H}+\sigma^{2} \mathbf{I}\right)^{-1} \mathbf{a}_{i}
$$

we arrive at $\mathbf{w}_{l}=s_{i}^{(l)} \hat{\mathbf{w}}$ for $l=1, \ldots, G$. At the output of the $l$ th combiner, the resulting statistic can be expressed as

$$
\hat{y}_{l}=\mathbf{w}_{l}^{H} \mathbf{r}_{l}=\sum_{j=1}^{N} \sqrt{p_{j}} b_{j} \hat{\mathbf{w}}^{H} \mathbf{a}_{j} s_{i}^{(l)} s_{j}^{(l)}+\hat{n}_{l}
$$

where $\hat{n}_{l}=\mathbf{w}_{l}^{H} \mathbf{n}_{l}$. Defining $\hat{s}_{j}^{(l)}=\hat{\mathbf{w}}^{H} \mathbf{a}_{j} s_{i}^{(l)} s_{j}^{(l)}$, we have

$$
\hat{y}_{l}=\sum_{j=1}^{N} \sqrt{p_{j}} b_{j} \hat{s}_{j}^{(l)}+\hat{n}_{l}, \quad l=1, \ldots, G
$$

It remains to find the MMSE combiner for $\hat{\mathbf{y}}$. We can express this second stage of the cascaded filter as

$$
\mathbf{c}=\sqrt{p_{i}}\left(\sum_{j=1}^{N} p_{j} \hat{\mathbf{s}}_{j} \hat{\mathbf{s}}_{j}^{H}+\frac{1}{G} \sigma^{2}\left(\hat{\mathbf{w}}^{H} \hat{\mathbf{w}}\right) \mathbf{I}\right)^{-1} \hat{\mathbf{s}}_{i}
$$

Notice that, as in the case of Section 3.4 , the noise covariance matrix is given by $\operatorname{diag}\left\{\sigma^{2}\left(\mathbf{w}_{l}^{T} \mathbf{w}_{l}\right)\right\}$. Since $\mathbf{w}_{l}^{H} \mathbf{w}_{l}=$ $(1 / G)\left(\hat{\mathbf{w}}^{H} \hat{\mathbf{w}}\right)$ for all $l$, the noise covariance matrix reduces to $\left(\sigma^{2} / G\right)\left(\hat{\mathbf{w}}^{H} \hat{\mathbf{w}}\right) \mathbf{I}$. The final bit decision is done by taking the sign of the real part of $y_{i}=\mathbf{c}^{H} \hat{\mathbf{y}}$.

Note that to construct the overall receiver we need to invert one $K \times K$ matrix to calculate $\hat{w}$ and one $G \times G$ matrix to calculate $c$. To see how the overall cascaded filter can be expressed as a matrix filter $\mathbf{X}_{i}^{\text {ST-CMMSE }}$, observe that

$$
\hat{y}_{l}=\mathbf{e}_{l}^{\top} \mathbf{R} \mathbf{w}_{l}^{*}=s_{i}^{(l)} \operatorname{tr}\left(\hat{\mathbf{w}}^{*} \mathbf{e}_{l}^{\top} \mathbf{R}\right)
$$

and

$$
y_{i}=\sum_{l=1}^{G} c_{l}^{*} \hat{y}_{l}=\operatorname{tr}\left(\hat{\mathbf{w}}^{*}\left(\sum_{l=1}^{G} c_{l}^{*} s_{i}^{(l)} \mathbf{e}_{l}^{\top}\right) \mathbf{R}\right)
$$

Then, comparing (25) with (6), we find that

$$
\mathbf{X}_{i}^{\mathrm{ST}-\mathrm{CMMSE}}=\left(\sum_{l=1}^{G} c_{l} s_{i}^{(l)} \mathbf{e}_{l}\right) \hat{\mathbf{w}}^{\top}
$$

which is of rank 1 .

\subsection{Optimum Temporal-Spatial MMSE Filter}

The optimum matrix filter in temporal and spatial domains which minimizes the MSE between $y_{i}$ and $b_{i}$ is

$$
\mathbf{X}_{i}^{\mathrm{O}-\mathrm{MMSE}}=\arg \min _{\mathbf{X}} E\left[\left|\boldsymbol{t r}\left(\mathbf{X}^{H} \mathbf{R}\right)-b_{i}\right|^{2}\right]
$$

The optimization problem (27) can be converted to an optimization problem with vector variables for easier manipulation [11]. The problem then becomes a straightforward extension of the standard MMSE problem. Its solution is $[6,7,11,13,15]$ :

$$
\mathbf{x}_{i}=\sqrt{p_{i}}\left(\sum_{j=1}^{N} p_{j} \mathbf{q}_{j} \mathbf{q}_{j}^{H}+\sigma^{2} \mathbf{I}\right)^{-1} \mathbf{q}_{i}
$$

where $\mathrm{q}_{j}$ is the temporal-spatial signature of user $j$ and is constructed by stacking columns of $s_{j} \mathbf{a}_{j}^{\top}$ as a long vector of size $K G$. The matrix filter $\mathbf{X}_{i}^{\text {O-MMSE }}$ is constructed by taking every $G$ elements of $\mathbf{x}_{i}$ and putting as a column to $\mathrm{X}_{i}^{\mathrm{O}-\mathrm{MMSE}}$.

The joint MMSE filter requires a possibly large matrix $(K G \times K G)$ to be inverted which can be computationally costly, or the corresponding adaptive implementation may be slow. This is the reason why we consider the less complex joint MMSE filter in the next section.

\subsection{Constrained Optimum Temporal-Spatial MMSE Filter}

To reduce complexity of the temporal and spatial filtering with little sacrifice in performance, $[15,16]$ proposed finding the optimum matrix filter in a constrained class of matrix filters. The proposed constrained class is rank 1 matrix filters, or the separable temporal-spatial filters, i.e., the filters that can be expressed as $\mathbf{X}_{i}=\mathrm{cw}^{\top}$. We can find the joint optimal filter pair in the MMSE sense for this constrained class. The optimization problem in (27) becomes

$$
[\overline{\mathbf{c}}, \overline{\mathbf{w}}]=\arg \min _{\mathbf{c}, \mathbf{w}} E\left[\left|\mathbf{c}^{H} \mathbf{R} \mathbf{w}^{*}-b_{i}\right|^{2}\right]
$$

The resulting $[\overline{\mathbf{c}}, \overline{\mathbf{w}}]$ pair yields the matrix filter

$$
\mathrm{X}_{i}^{\text {CO-MMSE }}=\overline{\mathbf{c}} \overline{\mathbf{w}}^{\top}
$$

This matrix filter is suboptimal for the optimization problem given in (27) since it is found in a constrained $\mathrm{X}_{i}$ space. The MSE function in (29) can be expressed as

$$
\begin{aligned}
\text { MSE }= & \sum_{j=1}^{N} p_{j}\left|\mathbf{c}^{H} \mathbf{s}_{j}\right|^{2}\left|\mathbf{w}^{H} \mathbf{a}_{j}\right|^{2}+\sigma^{2}\left(\mathbf{c}^{H} \mathbf{c}\right)\left(\mathbf{w}^{H} \mathbf{w}\right) \\
& -2 \sqrt{p_{i}} \Re\left\{\left(\mathbf{c}^{H} \mathbf{s}_{i}\right)\left(\mathbf{w}^{H} \mathbf{a}_{i}\right)\right\}+1
\end{aligned}
$$

where $\Re\{\cdot\}$ denotes the real part of a complex number. The minimizer of (31) does not have a closed form expression [15]. Further, the MSE function is not jointly convex in $\mathrm{c}$ and $w$, although it is convex in each variable ( $c$, or $w)$, when the other variable is fixed. Thus, standard iterative optimization algorithms cannot guarantee convergence to global minima. However, an alternating minimization algorithm 
[3] is given in [15] that is observed to have good convergence properties. We restate the algorithm here for convenience.

Consider fixing the value of one of the filters; say $w$ is fixed to $\tilde{w}$. It is then possible to find the filter, $\hat{c}$, that maximally decreases the MSE function in (31). The solution is analogous to the MMSE detector [6], where user $j$ 's received amplitude is modified such that it is $\sqrt{p_{j}}\left(\tilde{\mathbf{w}}^{H} \mathbf{a}_{j}\right)$. Denote this filter as $\hat{\mathbf{c}}=\operatorname{MMSE}(\tilde{\mathbf{w}})$ :

$$
\hat{\mathbf{c}}=\sqrt{p_{i}}\left(\tilde{\mathbf{w}}^{H} \mathbf{a}_{i}\right)\left(\sum_{j=1}^{N} p_{j}\left|\tilde{\mathbf{w}}^{H} \mathbf{a}_{j}\right|^{2} \mathbf{s}_{j} \mathbf{s}_{j}^{H}+\sigma^{2}|\tilde{\mathbf{w}}|^{2} \mathbf{I}\right)^{-1} \mathbf{s}_{i}
$$

The same argument can be made for the case where $c$ is fixed to $\tilde{\mathrm{c}}$ and the spatial filter is found to maximally decrease the $\operatorname{MSE}, \hat{\mathbf{w}}=\operatorname{MMSE}(\tilde{\mathbf{c}})$ :

$$
\hat{\mathbf{w}}=\sqrt{p_{i}}\left(\tilde{\mathbf{c}}^{H} \mathbf{s}_{i}\right)\left(\sum_{j=1}^{N} p_{j}\left|\tilde{\mathbf{c}}^{H} \mathbf{s}_{j}\right|^{2} \mathbf{a}_{j} \mathbf{a}_{j}^{H}+\sigma^{2}|\tilde{\mathbf{c}}|^{2} \mathbf{I}\right)^{-1} \mathbf{a}_{i}
$$

Now, consider the following algorithm. Starting with the filter pair $c(0), w(0)$ and keeping $\mathbf{w}(0)$ fixed, one can find $\mathbf{c}(1)=\operatorname{MMSE}(\mathbf{w}(0))$. This operation decreases the MSE defined in (31). Then keeping $\mathbf{c}(1)$ fixed, one can find $\mathbf{w}(1)=\operatorname{MMSE}(\mathbf{c}(1))$. This operation further decreases the MSE in (31). Iteration $n+1$ of this two step iterative algorithm for user $i$ is given below.

$$
\begin{aligned}
\mathbf{c}(n+1) & =\operatorname{MMSE}(\mathbf{w}(n)) \\
\mathbf{w}(n+1) & =\operatorname{MMSE}(\mathbf{c}(n+1))
\end{aligned}
$$

Note that the order in which $c$ and $w$ are updated could be reversed. After each two-step iteration given by (34) and (35), the MSE in (31) monotonically decreases. The algorithm is provably convergent and the convergence point is experimentally observed to be the optimum pair $[\overline{\mathbf{c}}, \overline{\mathbf{w}}]$ where the MSE is minimized and the SIR of the user is maximized [15].

\section{Performance Comparison}

An important performance comparison criterion is the bit error rate (BER). Unfortunately, for general system parameters, it is difficult to derive analytical results for the BER rendering this comparison intractable. Commonly the BER is expressed as a function of the SIR by applying a Gaussian approximation to the total interference. It was reported in [10] that this approximation is particularly accurate when MMSE receivers are employed. For a general matrix filter $\mathrm{X}$, the MSE and the SIR are related as (for details, see the
Appendix of [15])

$$
\frac{1}{\min _{\alpha} \operatorname{MSE}(\alpha \mathbf{X})}=1+\operatorname{SIR}(\mathbf{X})
$$

Thus, with an appropriate scaling, the MSE and the SIR produced by a filter can be related, and the filter that minimizes the MSE also maximizes the SIR. Note that the SIR, and therefore the BER when defined in terms of the SIR, are insensitive to the scaling of the linear receiver filter.

From the arguments above, it is clear that the optimum MMSE receiver of Section 3.6 outperforms all other receiver structures mentioned in Sections 3.1 through 3.5, as well as the constrained optimum MMSE receiver in Section 3.7, in terms of both the MSE and the SIR. The reason for this is that the filter in Section 3.6 is chosen to minimize the MSE over all possible matrix filters. It only remains to compare the performance of the constrained optimum MMSE receiver of Section 3.7 with the receiver structures in Sections 3.1 through 3.5. First we observe from (7), (8), (10), (26) that the filters $\mathrm{X}_{i}^{\mathrm{MF}-\mathrm{MF}}, \mathrm{X}_{i}^{\mathrm{MMSE}-\mathrm{MF}}, \mathrm{X}_{i}^{\mathrm{MF}-\mathrm{MMSE}}$, $\mathrm{X}_{i}^{\text {ST-CMMSE }}$ are of rank 1 . Given that the constrained optimum MMSE, $X_{i}^{\text {CO-MMSE }}$, minimizes the MSE and therefore maximizes the SIR among all possible rank 1 matrix filters, we conclude that the constrained optimum MMSE receiver filter outperforms all of these suboptimum receiver filters. In fact, the iterative algorithm described by ((34),(35)) can be started at any of the temporal-spatial filter pairs that define $\mathbf{X}_{i}^{\mathrm{MF}-\mathrm{MF}}, \mathbf{X}_{i}^{\mathrm{MMSE}-\mathrm{MF}}, \mathbf{X}_{i}^{\mathrm{MF}-\mathrm{MMSE}}$ or $\mathbf{X}_{i}^{\mathrm{ST} \text {-CMMSE}}$. Since each iteration increases the SIR and decreases the MSE monotonically, with each iteration, the performance of the resulting filter pair is better than the previous one and the convergence point temporal-spatial filter pair, $\mathbf{X}_{i}^{\text {CO-MMSE }}$, outperforms the starting point filter pair.

The cascaded temporal-spatial MMSE filter, $\mathbf{X}_{i}^{\text {TS-CMMSE, }}$ in Section 3.4 can have rank up to $K$, just as the joint optimum MMSE filter of Section 3.6 which can have rank up to $\min \{K, G\}$. Thus, there could be cases under which $\mathbf{X}_{i}^{\text {TS-CMMSE }}$ performs better than the constrained optimum filter; see Section 5. However, the fact that $\mathbf{X}_{i}^{\text {TS.CMMSE }}$ has higher rank than $\mathbf{X}_{i}^{\text {CO-MMSE }}$ does not necessarily guarantee that it yields a lower MSE or a higher SIR than

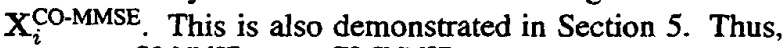
neither $\mathbf{X}_{i}^{\text {CO-MMSE }}$ nor $\mathbf{X}_{i}^{\text {TS-CMMSE }}$ performs uniformly better than the other; depending on the system parameters $(N$, $G, K$, spatial and temporal signatures of the users, etc.) one may outperform the other.

It is possible to compare the two filters in the asymptotic regime when the background noise $\sigma^{2}$ goes to zero, or equivalently the received powers of the interfering users go to infinity. It is well-known that the MMSE receiver reduces to a decorrelating receiver as the background noise power goes to zero or the received powers of the interfering users go to infinity [12]. The decorrelating receiver [5] is a 
linear multiuser detector which suppresses the multiaccess interference totally. This is done by projecting the desired user's signal onto the subspace that is orthogonal to the signal space spanned by the interfering users. The decorrelation operation is independent of the received powers of the users and only depends on their signature sequences. The multiaccess interference is suppressed totally if the desired user's signature sequence is linearly independent of the interfering signatures. Nevertheless, the decorrelating detector exists even when the signature sequences of the users are not linearly independent; in this case one needs to use the Moore-Penrose generalized inverse of the cross correlation matrix as opposed to its direct inverse [5]. The decorrelator is independent of the received powers in this case as well [5].

Recall now that in the calculation of the cascaded temporal-spatial MMSE receiver filter $\mathbf{X}_{i}^{\text {TS-CMMSE }}$, first, $K$ temporal MMSE receiver filters are found and that these $K$ MMSE receivers, $c_{k}$ 's, are different due to the fact that the received powers of the users are different at each antenna array element; see (13). This is because the actual received powers of the users are multiplied with the square magnitudes of the antenna gains $\left|a_{j}^{(k)}\right|$ at the outputs of different antenna elements. Since in the interference limited regime the MMSE receivers go to decorrelators and since decorrelators are independent of the received powers of the users, all $K$ temporal receiver filters become identical, i.e., $\mathbf{c}_{k}=\mathbf{c}$ for all $k$. Note that this is true even when the cross correlation matrix is not invertible and the Moore-Penrose generalized inverse is used. When the temporal filters at the output of all antenna array elements are the same, the receiver filter $X_{i}^{\text {TS-CMMSE }}$ becomes a rank 1 filter. The MSE achieved by $\mathrm{X}_{i}^{\mathrm{TS}-\mathrm{CMMSE}}, \mathrm{MSE}_{i}^{\mathrm{TS}-\mathrm{CMMSE}}$, is larger than that of the constrained optimum MMSE filter, $\mathrm{MSE}_{i}^{\mathrm{CO}-\mathrm{MMSE}}$, simply because $\mathrm{X}_{i}^{\mathrm{CO}-\mathrm{MMSE}}$ is the filter that yields the minimum MSE among all rank 1 matrix filters. Thus,

$$
\lim _{\sigma^{2} \rightarrow 0} \mathrm{MSE}_{i}^{\mathrm{TS}-\mathrm{CMMSE}} \geq \lim _{\sigma^{2} \rightarrow 0} \mathrm{MSE}_{i}^{\mathrm{CO}-\mathrm{MMSE}}
$$

Equivalently, using (36), the SIRs achieved by these two filters in this interference limited regime is compared as

$$
\lim _{\sigma^{2} \rightarrow 0} \operatorname{SIR}_{i}^{T S-C M M S E} \leq \lim _{\sigma^{2} \rightarrow 0} \operatorname{SIR}_{i}^{\text {CO-MMSE }}
$$

Hence, the constrained optimum MMSE filter outperforms the cascaded temporal-spatial MMSE filter of Section 3.4 asymptotically.

\section{Results and Conclusions}

We consider a single cell CDMA system, the base station of which employs a linear antenna array [13]. The temporal signatures and users' positions which in turn determine the spatial signatures are created randomly, and kept fixed for

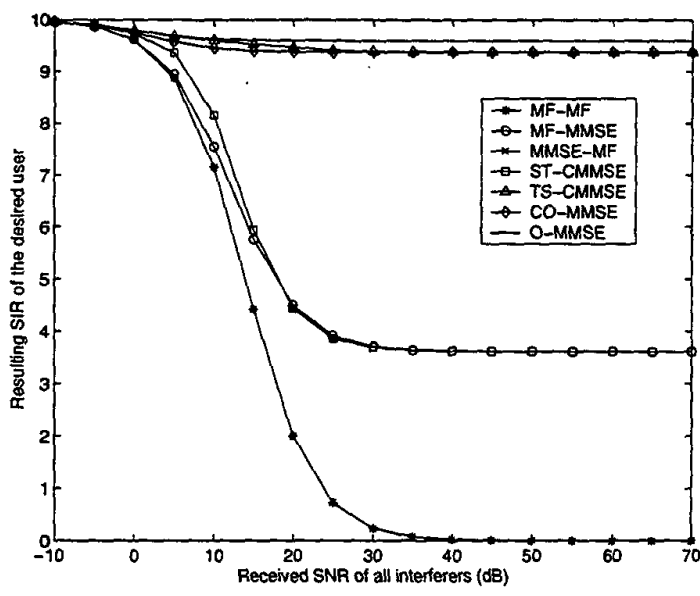

Figure 1. $N=2, K=2, G=8$.

the duration of the experiment. We plot the output signal-tointerference ratio (SIR) for the desired user (in linear scale) versus the received signal-to-noise ratios (SNRs) of all interferers (in $\mathrm{dB}$ scale). The desired user's SNR is $10 \mathrm{~dB}$.

Consider first a system with processing gain $G=8$, $K=2$ array elements and $N=2$ users. Figure 1 shows the output SIR of the desired user. As expected, with only a single interferer present, all filters perform well even under very severe near-far conditions where the interferer's power is as much as $60 \mathrm{~dB}$ above the desired user's. The only exception is the temporal-spatial matched filter which is wellknown to be not near-far resistant. The more interesting observation about this system is better observed in Figure 2. Recall that we concluded in Section 4 that the constrained optimum MMSE filter does not necessarily outperform the cascaded temporal-spatial MMSE filter of Section 3.4, it only is as good or better than the cascaded temporal-spatial MMSE filter asymptotically. Indeed, in Figure 2, we see that the cascaded temporal-spatial MMSE outperforms the constrained optimum MMSE filter. When the system becomes interference limited, both detectors have identical performance. The constrained optimum MMSE filter outperforms all other filters, except for the optimum MMSE filter which is the SIR maximizer among all matrix filters.

For the rest of this section, the system considered has $\dot{K}=4$ antenna array elements and $G=16$ processing gain. We will examine the performance of the filter structures for this system under different loading conditions.

We first consider $N=8$ users. Figure 3 shows the output SIR of the desired user. The constrained optimum MMSE filter outperforms all filters, except for the optimum MMSE filter. Note that, for this system, user's temporal signature sequences are linearly independent and thus, when the system is interference limited, the filters that perform 


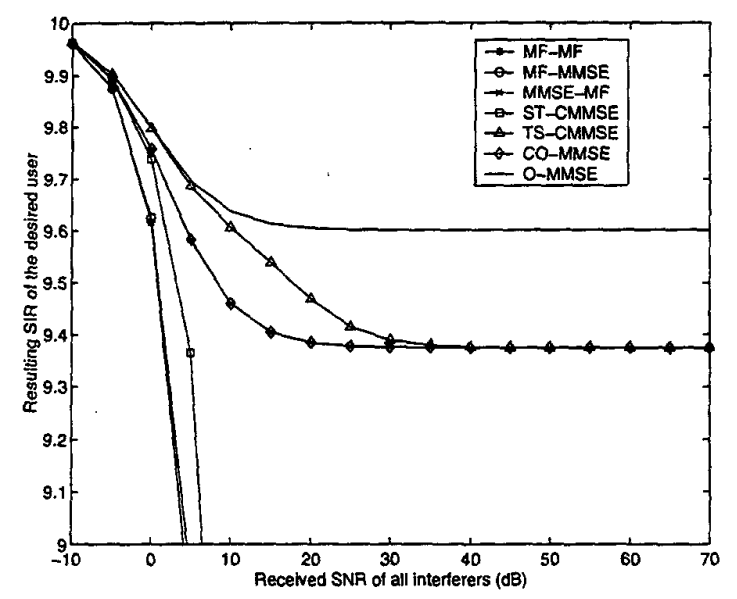

Figure 2. Figure 1 magnified.

temporal MMSE first, i.e., the temporal MMSE filter-spatial matched filter of Section 3.3 and the cascaded temporalspatial MMSE filter of Section 3.4 end up decorrelating all interferers in temporal domain. Specifically, the temporal MMSE filter at the output of each antenna becomes a decorrelator, $c_{k}=\mathbf{c}$, for all $k=1, \ldots, K$, for the the cascaded temporal-spatial MMSE filter. In this case, the output statistics of the first stage of the cascaded temporal-spatial MMSE filter are interference free, i.e., (14) can be expressed as

$$
\tilde{\mathbf{y}}=\sqrt{p_{i}} b_{i}\left(\mathbf{c}^{H} \mathbf{s}_{i}\right) \mathbf{a}_{i}+\tilde{\mathbf{n}}
$$

where $\tilde{n}_{k}$ is the enhanced noise at the output of the $k$ th antenna and the components of $\tilde{\mathbf{n}}$ are independent. Thus, the second stage spatial MMSE combiner $w$ in (15) becomes the spatial matched filter, $\mathbf{a}_{i}$, which explains why the temporal MMSE-spatial matched filter and the cascaded temporalspatial MMSE filter have identical performance asymptotically. It is also notable that constrained optimum MMSE detector becomes a temporal-spatial decorrelator and chooses to suppress some of the interferers in temporal domain and others in spatial domain such that it gets the best asymptotic SIR among such temporal-spatial decorrelators.

Next we consider $N=16$ users. The output SIR of the desired user is plotted in Figure 4. There are still enough temporal dimensions for users to be decorrelated in the temporal domain, i.e., $N \leq G$, even if they can not all be suppressed in the spatial domain. As a result, all filters that employ MMSE combining in the temporal domain have nonzero SIRs asymptotically. However, the constrained optimum MMSE filter, by choosing the appropriate users to suppress in the spatial or temporal domains, achieves higher SIR over all filters except the optimum MMSE filter.

The next system to be considered has $N=18$ users and results are shown in Figure 5. Since the number of interferers are larger than both the processing gain and the number

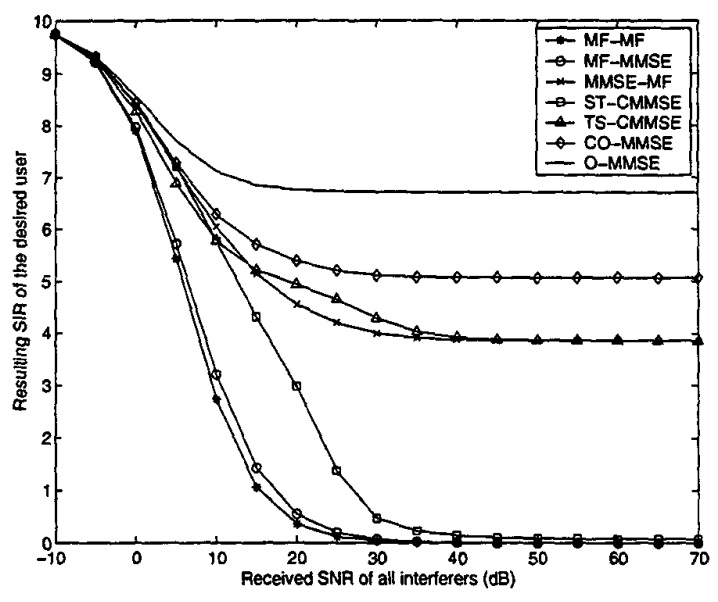

Figure 3. $N=8, K=4, G=16$.

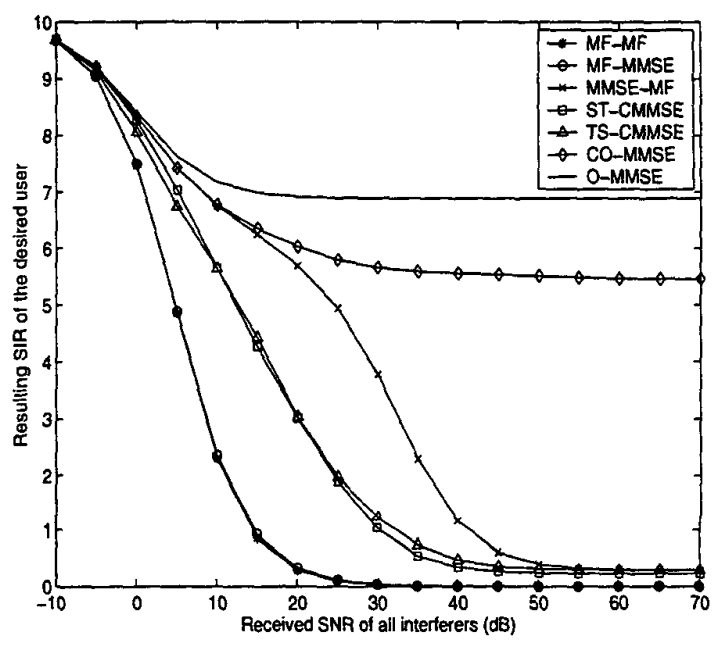

Figure 4. $N=16, K=4, G=16$.

of array elements, all interferers cannot be suppressed in a single domain, thus the combined single user/multiple user

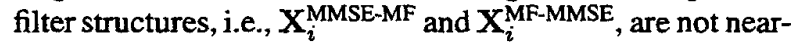
far resistant. For this example, the cascaded structures, i.e.,

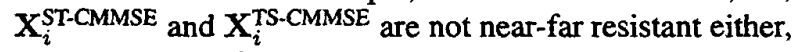
since in the interference dominated regime, each stage tries to suppress all interferers independently in cascade. When an interference suppressor is designed by considering both domains jointly, as in the case of constrained optimum and optimum detectors, $\mathbf{X}_{i}^{\text {CO-MMSE}}$, and $\mathbf{X}_{i}^{\mathbf{O}-M M S E}$, near-far resistance is achieved.

The last example we consider is a very highly loaded system with $N=36$ users. The purpose of this experiment is to show the difference between the optimum MMSE filter and the constrained optimum MMSE filter. We observe from 


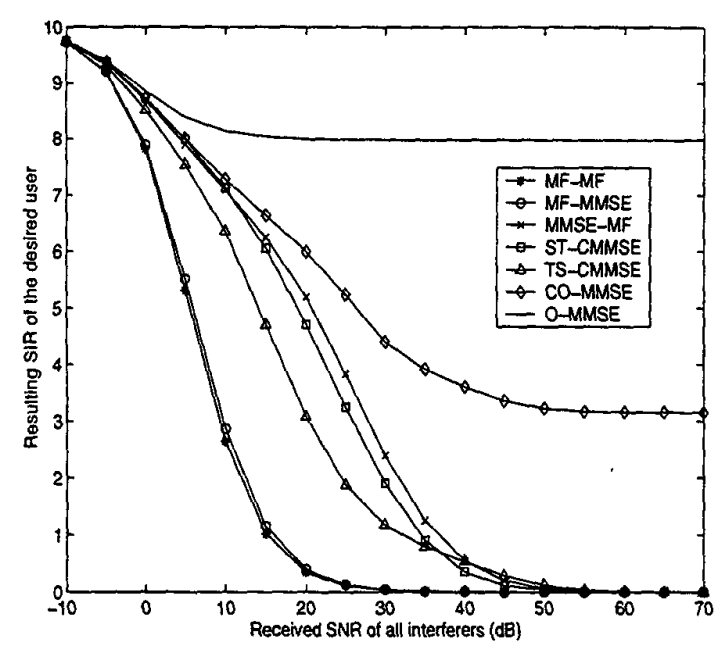

Figure 5. $N=18, K=4, G=16$.

Figure 6 that although the constrained optimum MMSE filter, $\mathbf{X}_{i}^{\text {CO-MMSE }}$, results in acceptable SIR values in near-far situations, e.g. an output SIR of $5(7 \mathrm{~dB})$ when all interferers' powers are $10 \mathrm{~dB}$ higher than the desired user, it is not near-far resistant. This is simply due to the fact that the constrained optimum MMSE filter can suppress up to $G-1$ users in the temporal domain and $K-1$ users in the spatial domain. Thus, for this example, when $N>19$, the constrained optimum MMSE filter is not able to suppress all the interference and the output SIR it produces approaches 0 when the interferers' powers approach infinity. The optimum MMSE detector on the other hand can suppress up to $K G-1$ users and for this example is near-far resistant.

\section{References}

[1] F. Adachi, M. Sawahashi, and H. Suda. Wideband DSCDMA for next-generation mobile communication systems. IEEE Comm. Mag., 36(9):56-69, September 1998.

[2] X. Bernstein and A. M. Haimovich. Space-time optimum combining for CDMA communications. Wireless Personal Communications, (3):73-89, 1996.

[3] J. A. Fessler and A. O. Hero. Space-alternating generalized expectation-maximization algorithm. IEEE Trans. Signal Proc., 42(10):2664-2677, October 1994.

[4] R. Kohno, H. Imai, M. Hatori, and S. Pasupathy. Combination of an adaptive array antenna and a canceller of interference for direct-sequence spread-spectrum multiple-access system. IEEE JSAC, 8(4):675-682, May 1990.

[5] R. Lupas and S. Verdú. Linear multiuser detectors for synchronous code-division multiple-access channels. IEEE Trans. Info. The., 35(1):123-136, January 1989.

[6] U. Madhow and M. L. Honig. MMSE interference suppression for direct-sequence spread-spectrum CDMA. IEEE Trans. Comm., 42(12):3178-3188, December 1994.

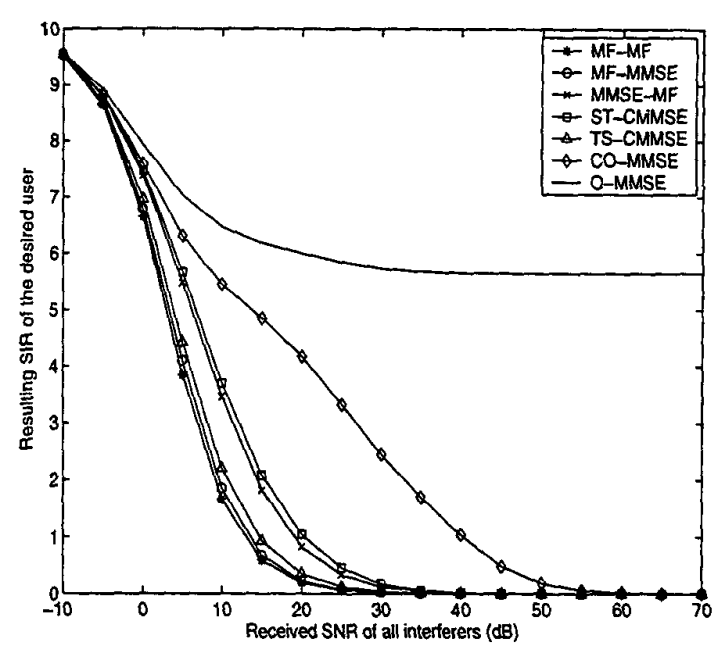

Figure 6. $N=36, K=4, G=16$.

[7] R. A. Monzingo and T. W. Miller. Introduction to Adaptive Arrays. Wiley, 1980.

[8] A. F. Naguib, A. J. Paulraj, and T. Kailath. Capacity improvement with base-station antenna arrays in cellular CDMA. IEEE Trans. Vehic. Tech., 43(3):691-698, August 1994.

[9] A. J. Paulraj and C. B. Papadias. Space-time processing for wireless communications. IEEE Signal Proc. Mag., 14(6):49-83, Novernber 1997.

[10] H. V. Poor and S. Verdú. Probability of error in MMSE multiuser detection. IEEE Trans. Info. The., 43(3):858-871, May 1997.

[11] V. G. Subramanian and U. Madhow. Blind demodulation of direct-sequence CDMA signals using an antenna array. In CISS, 1996.

[12] S. Verdú. Multiuser Detection. Cambridge University Press, 1998.

[13] X. Wang and H. V. Poor. Space-time multiuser detection in multipath CDMA channels. IEEE Trans. Signal Proc., 47(9):2356-2374, September 1999.

[14] T. Wong, T. M. Lok, J. S. Lehnert, and M. D. Zoltowski. A linear receiver for direct-sequence spread-spectrum multiple-access systems with antenna arrays and blind adaptation. IEEE Trans. Info. The., 44(2):659-676, March 1998.

[15] A. Yener, R. D. Yates, and S. Ulukus. Interference management for CDMA systems through power control, multiuser detection, and beamforming. IEEE Trans. Comm., 1999. Submitted. http://www. winlab.rutgers.edu/ yener.

[16] A. Yener, R. D. Yates, and S. Ulukus. Joint power control, multiuser detection and beamforming for CDMA systems. In IEEE VTC, May 1999. 